\title{
Muerte cerebral neonatal: reflexiones a partir de un caso clínico
}

\author{
Juan Pablo Beca I. ${ }^{1}$; María Teresa Rotondo T. ${ }^{2}$
}

\begin{abstract}
Resumen
:a muente cerebrcl continúa siendo un teme ce débale cullural y secial. En el caso de los recién racidos no se ha concordado una definicióri claro, to que lleva a mayores confusones. Se describe el coso clínico de tno niño en la cual se diagnos icó muerte cerebral, y las dificultades médicas y fomiliares implicitas. El coso es comentacio oor un necráólogo y ura neviólogo pediátrico en sus aspectos bicélicos, analizondo las conciciones para un diagnóstico de certezc, los conceptos de muente, las cecisiones de ctsiención o suspensión teropéutica, y la necesidad de relación méd co-sociente bascda en lo contianza.
\end{abstract}

[Palabras clave: ruere cerebral, recién nacido.]

\section{Brain death in a newborn infant}

The subject of brain death is $i^{-}$sermanen social and cullural debale. in spile of recise egal and medical definifions for adults ar.d oider crildren. In the case of newborr pci enis agreemen has not been reached for a cear orain decin definition, and herefore more con'usion is bllowed. A case of brain death in a newborn patient is presentes with its difficulties both ir medical and icmily perspectives. A neonatologist and a pediaric rieuro'ogist comment this case in a boethical perspective, discussing the requirements for an accurate diagnosis, the contepls of death, the decissions of foregoing or withrdrawing treatments, and the rieed for a potien-iprysicicn relationship based on trust.

(Koy words: broin death, newborn.)

Entre los difíciles temas que hoy enfrenta la bjoética está el de la muerte cerebral, sus definiciones y sus repercusiones filosóficas, religiosas, sociales y legales. La literatura en relación con el tema es amplia y, aunque las legislaciones de casi todos los países reconocen esta situación y asumen definiciones legales, aún persiste el debate entre los especialistas y en toda la sociedad.

Pero en el caso de los recién nacidos la muerte cerebral ocupa menos espacio en las publica-

1. Unidad de Neonatología Clínica Alemana de Santiago. Facultad de Medicina Universidad de Chile. Programa Regional de Bioética OPS/OMS.

2. Instituto de Neurología, Facultad de Medicina, Universidad de la República, Departamento Neuropediatría Hospital Policial de Montevideo, Comisión de Bioética del Sindicato Médico del Uruguay. ciones y su diagnóstico, así como las decisiones médicas y familiares que le siguen plantean importantes dudas y a veces conflictos de difícil solución. Con el propósito de contribuir a aclarar conceptos sobre este tema entre los pediatras, se presenta un caso clínico, las decisiones médicas y familiares que se tomaron, y los comentarios independientes, desde el punto de vista ético, de un neonatólogo y un neurólogo-pediatra.

\section{Caso clínico}

Recién nacida de 40 semanas de gestación, cuarto hijo de padres sanos, embarazo controlado sin enfermedades, parto vaginal, trabajo de parto vigilado con registros fetales normales. Perrodo expulsivo de aproximadamente 20 minutos. Al nacer là niña estaba profundamente deprimida. sin actividad propia. El Apgar fue $\mathrm{I}$ al minuto con frecuencin cardíaca 60 por minuto. Se le practicó intubación tra- 
queal, lográndose buena expansión pulmonac y recuperación de la frecuencia cardíaca A los 5 minutos estaba rosada pero con bipotonia, sin respuesta refleja y movimientos respiratorios. Fue llevada a cuidados intensivos y conectada a ventilador mecánico para mantener saturación normal de oxígeno en la sangre. Tenía acidosis metabólica severa ( $\mathrm{pH} 7.10$ y exceso de base (BE) $-19.5 \mathrm{mmol} / 1$ a las 3 horas de edad), ácido láctico 120 y $123 \mathrm{mg} \%(\mathrm{~N}=5-15)$, amonio normal, troponina $T 6,3(N=0,1-3)$. creatininguinasa MB $86 \mathrm{U} / \mathrm{l}(\mathrm{N}=\mathbf{0}-10)$. mioglobina $\mid 6] \mathrm{ng} / \mathrm{ml}$ $\left(N^{\prime}=16-76\right)$ en el suero. Líquido cefaloraquídeo (LCR) y ultrasonografía cerebral (USC) normales. La tomograffa axial computadorizada (TAC) mostró imagen sospechosa de hetnorragia bulbar. descartada mediante resonancia nuclear magnétjca (RNM). que sólo mostró signos đe edema cerebral. La paciente no presentó actividad propia, salvo algunas descargas motrices sospechasas de movimientos convulsivos a las 24 horas después de nacer. Fue tratada con ventilación mecánica, corrección de la acidosis metabólica inicial, apoyo con drogas vasoactivas hasta obrener diuresis y estabilización de presión arterial al segundo día. y fenobarbital como anticonvulsivo solo por 24 horas. En el primer etectroencefalograma (EEG), al segundo día de vida, se registró marcada disminución de voliaje y ocasionales paroxismos, compatibles con severa encefalopatía difusa, sin actividad epileptifome. Evoluciono con total itnacrividad y ausencia de respuesta a estímulos. estableciéndose midriasis paralitica al tercer dra de vida. El registro del segundo EEG era plano, compatible con el diagnóstico de muerte cerebral.

Al tercer día de vida su padre manifestó la voluntad de "no hacer nada extraordinario", rechazando inicialmente la RNM para confirmar el diagnóstico de hemorzagia. Dada su mala evolución y el planteamiento diagnóstico de muerte cerebral, el padre manifestó yerbalmente su voluntad de desconectar a la niña del respirador, pero solicitó no asumir esta responsabilidad por escrito. Se convino en que firmase una autorización para suspender la ventilación mecánica, pero sólo después de confirmar el diagnóstico de muerte cerebral mediante otro EEG y una prueba de apnea. Al cuarto día de vida, en el tercer EEG, se confirmó el registro plano $y$ la prueba de apnea fue seguida por la aparición de respiración espontánea en forma de boqueadas ("gasping") con frecuencias de 10 a 15 por minuto y registro estable del electrocardiograma (ECG), razón por la cual se decidió proponer al padre mantener con iubo endutraqueal y la presión positiva de $3 \mathrm{~cm} \mathrm{H}_{2} \mathrm{O}$, sin ciclar el respirador y sin realizar más estudio diagnóstico ni nuevos intentos terapéuticos. Se tirmó consentimiento en este senlide, sin la presencia de la madre. Se insisció en que la madre $y$ los hermanos, de 11 a 7 años de edad, visitasen $y$ acompañasen a la niña en esta etapa fınal, hecho que el padre no consideró conveniente. La niña fue visitada varias veces por el padre, la madre la visito breves minutos en una oportunidad, y recibió una vez la visita de una tía y de la abuela paterna. Falleció 36 h después, al sexto día de vida, sin orta manifestación clínica que progresivos períodos de bradicardia en ausencia de sus familiares.

Las decisiones terapéticas se tornaron cn reuniones de tres o más neonatólogos, contando además con el aporte de la opinión de un neurólogo. Además se analizo detalladamente el caso con el obstetra. indagando por la causa de la hipoxia profunda, que al parecer ocurrio durante el período expulsivo. En las visitas con personal de enfermería, y en reuniones con cada turno de auxiliares, se les explicó en detalle el caso y las razones de las actitudes asumidas

\section{Comentario}

Juan Pablo Beca: Este caso presenta, desde el punto de vista ético, varios aspectos de interés. Sin embargo en este comentario nos referiremos solamente a la complejidad del diagnóstico de muerte cerebral neonatal a algunos aspectos de la relación médico-paciente y la toma de decisión.

El concepto de muerte cerebral sigue siendo un tema en discusión. En él está involucrada la aceptación de la muerte de la corteza cerebral, y para algunos necesariamente de todo el encéfalo, como equivalente a la desaparición de la persona. En este caso es legítima la suspensión de todo tratamiento, que sólo prolongaría la vida de algunos órganos o sistemas sin unidad, y también la extracción de los órganos que pueden ser empleados en trasplantes si conservan su vitalidad. Los signos clínicos que definen este estado o forma de muerte son convencionales y los criterios más aceptados aún son los de Harvard, que exigen: "no respuesta, no receptividad, ausencia de movimientos y respiración (necesidad de la prueba de apnea si el paciente está en ventilación mecánica), arreflexia, y EEG plano", en ausencia de hipotermia o depresión del SNC por otros factores'.

En caso de los reciên nacidos existe menor precisión conceptual. La mayor parte de la discusión se ha centrado en el estatuto moral de los anencefálicos como probables "donantes" de organos. El argumento en favor es que si la pérdida de funciones corticales se acepta como pérdida de la condición de persona, la ausencia de corteza, aunque exista función bulbar, se puede considerar una condición en la cual no se trata de personas vivas y, por lo tanto, se podría disponer de sus órganos. Los argumentos en contra son numerosos, basados en que se trata de malformados graves -no de personas que han perdido irreversiblemente sus funciones cerebralescuyo mal pronóstico vital y de desarrollo no es homologable a la muerte. En todo caso la situación de los anencefálicos es una particular, diferente, y requiere una fundamentación propia ${ }^{2-4}$. Cuando se ha planteado la muerte cerebral en 
niños recién nacidos, los criterios han sido menos precisos que los aplicados en adultos. Se requiere demostrar coma profundo, ausencia de reflejos craneanos y pruebas de apnea en todos los casos, recomendándose complementar el diagnóstico mediante EEG y estudios de flujo circulación sanguíneo cerebral. No siempre se realiza la prueba de apnea cuyo propósito es demostrar la pérdida de funciones de todo el encéfalo y no sólo de la corteza, pues la recuperabilidad de las funciones corticales a esta edad es impredecible $e^{5,6}$.

En el caso comentado el grupo de neonatólogos prefirió ser más conservador y estricto $y$, antes de plantear definitivamente este diagnóstico y sus conductas consecuentes, se practicó prueba de apnea que demostró la persistencia de actividad del centro respiratorio, por lo que se rechazó la solicitud de suspender la ventilación mecánica y se mantuvo a la paciente con tubo endotraqueal y presion positiva, hasta su muerte, horas después. Se plantea así, en un caso real, la validez del diagnóstico bien fundamentado y sus consecuencias. En caso de aceptar el diagnóstico neurológico y electroencefalográfico de muerte cerebral se podría haber considerado a la paciente como donante de órganos y procedido, previo consentimiento de sus padres, a su extracción. Probablemente nadie hubiese sabido que aún existía actividad bulbar y que la pérdida de funciones afectaba sólo a la actividad cortical. Pero cabe preguntarse si, en este caso, se hubiese extraído órganos a un cadáver o a un paciente vivo en estado vegetativo o en coma profundo. La respuesta sólo es posible mediante una clara definición de muerte cerebral neonital, que contemple indicadores biologicos y consideraciones culturales y filosóficas. Como ella no existe aún, parece prudente actuar en forma más conservadora. Esta conducta no conviene a los que esperan organos, pero no podenos responder a esos legítimos intereses con imprevisiones conceptuales o diagnósticas ventajosas. La falta de definiciones claras de muerte cerebral en el período neonatal, en países donde los recién nacidos son potenciales donantes de órganos, conduce con frecuencia a información incompleta o tendenciosa a los familiares de los eventuales donantes.

La excesiva "medicalización" de la muerte plantea nuevas interrogantes culturales, filosófjcas y religiosas que deben ser consideradas.
Aunque se adopte cultural y legalmente alguna definición de muerte cerebral, la aceptación de estas definiciones por parte de la sociedad o de las familias de los pacientes continúa siendo variable y muy probablemente seguirá cambian$\mathrm{do}^{\top}$. Así, ante una situación como la comentada, algunas familias pueden insistir en aplicar todo tipo de tratamiento, y otras en que se le considere fallecido y se le trate como tal. Se ha llegado a proponer que cada individuo o familia escoja su propia definición de muerte (cortical, encefálico o cardiorrespiratoria) de acuerdo a sus convicciones morales. Las complicaciones legales y sociales de este planteamiento son evidentes, pero de él se desprende la complejidad de un tema que estâ aún lejos de estar definitivamente aclarado ${ }^{8}$.

Otros aspectos importantes en este caso incluyen a la relación médico-paciente, cuyo elemento principal debe ser la confianza mutua. Las diferencias en el concepto de muerte entre el neurólogo, los neonatologos y el padre podrían haber causado gran desconfianza, lo que no ocurrí, probablemente debido a una buena relación previa entre obstetra y paciente, a la oportunidad, integridad y veracidad de la información, y a la comprensión y respeto por las actitudes familiares, aunque no coincidiesen con las recomendaciones de los médicos. La buena relación médico-paciente en casos penosos y de mal resultado es de beneficio para el paciente y la familia, que podrá elaborar su duelo sin una carga de dudas sobre el manejo médico y, secundariamente, para los médicos, que se verán libres de criticas o conflictos legales. La medicina instrumental, especializada, excesivamente institucionalizada o comercial, puede causar desconfianza en los médicos, dificultando una comunicación apropiada. Todo esfuerzo por mejorar las relaciones entre médicos, pacientes e instituciones de salud basadas en la confianza, serán de beneficio para todas las partes.

Así como la relación médico-paciente en el caso comentado fue de confianza recíproca, la reacción familiar fue confusa y diferente a la recomendada por el grupo médico tratante, más bien de rechazo y alejamiento, lo que es psicológicamente comprensible. Sin embargo, la negativa a asumir la situación produce dificultades para comprender los hechos y la elaboración familiar del duelo. Detrás de estas contradictorias reacciones familiares hay frecuentemente opi- 
niones de consejeros externos, pero en to fundamental está la confusión conceptual que la sociedad tiene sobre el final de la vida y su significado, tema en el cual médicos y demás profesionales de la salud deben tener claridad para poder apoyar adecuadamente a los pacientes.

Este caso es también un desafío a respetar la vida del recién nacido que está muriendo, a pesar de su mal pronóstico a corto y largo plazo. Dicho respeto significa aplicar tratamientos paliativos, calmar el dolor, otorgar apoyo psicológico y espiritual, y facilitar su contacto con la familia y aceptar, al mismo tiempo que validar $y$, muchas veces, admitir como el mejor camino, la muerte natural sin intervenir con medidas extraordinarias que podrían prolongar artificialmente la vida y el sufrimiento. Esta visión admite la legitimidad moral de suspender tratamientos, incluida la ventilación mecánica en algunos casos, pero de ninguna manera la de ninguna forma de producir la muerte o de extraer órganos antes de que ella se haya demostrado de acuerdo con los criterios médicos y legales vigentes.

Este caso, al igual que muchos mayor o menormente rutinarios de la práctica médica, plantea la difícil temática de cómo definir y decidir una conducta o decisión éticamente válida. Frecuentemente se buscan opiniones expertas, normas o formulas para encontrar una respuesta adecuada. Sin embargo se trata precisamente de situaciones en las cuales la toma de decisión exige un cuidadoso y profundo proceso de análisis. El estudio global de la situación en lo estrictamente médico junto al análisis de los aspectos valóricos y de los principios involucrados nos permiten liegar a conclusiones que, si están sólidamente fundamentadas, podremos considerar éticamente válidas. La étjca de la práctica médica es, en el fondo, un equilibrio entre una ética sustantivo o de principios, los métodos o procesos de toma de decisión, y la imprescindible ética de la conducta o de las virtudes profesionales ${ }^{9,10}$.

María Teresa Rotondo: El caso presentado ejemplifica una situación a la que frecuentemente se ven enfrentados los médicos desde hace varias décadas debido a los adelantos de las tecrologías, en especial en el campo de la neonatología. $Y$ nos muestra también las dificultades de establecer un diagnóstico certero acerca del verdadero estado clínico en los primeros siete días de vida, así como las dificultades del diagnóstico de muerte encefálica.

La impresión clínica ante este caso es que existió un severo sufrimiento encefálico predominantemente localizado en el tronco cerebral, como la que se observa en la interrupción de la circulación encefálico total y aguda.

Desde el establecimiento de los criterios de muerte cerebral se reconoce la dificultad de asegurarlos en la etapa neonatal de los siete primeros días de vida, debido a falta de suficiente informacion al respecto, aunque recientemente Ashwal estableció que es posible aplicarlos en ella" ${ }^{1}$. En casos especiales, cuando no se conoce la etiología, se exigen para la determinación de muerte cerebral otros estudios de la funcionalidad del tronco cerebral, como registro de potenciales evocados multimodales (que se modifican levemente por trastornos metabólicos endógenos) y el uso de reflejo de parpadeo. Antes de declarar el estado de muette encefálica se debe descartar que el niño sufra hipotermia. hipotensión o depresión por fármacos.

El problema que esta niña presentaba a los clínicos era determinar si el coma profundo era o no ineversible. Para resolverlo había que conocer la etiopatogenia con mayor precisión. Existe discusión en la literatura con respecto a la determinación de la muerte cerebral o encefálica (muerte cortical y del tronco cerebral) en los recién nacidos, sobre todo en la primera semana de vida y en los pretérminos. Los criterios establecidos para las edades superiores a la etapa neonatal precoz estipulan los siguientes requisitos: coma y apnea; ausencia de función del tronco cerebral definida como ausencia de reflejos oculomotores, fotomotor, corneano y movimientos respiratorios, incluyendo la prueba de apnea; descartar hipotensión e hipotermia; hipotonía y ausencia de movimientos, excepto los de origen medular; resultados del examen que correspondan a muerte cerebral durante todo el periodo de observación y pruebas ${ }^{12}$.

El diagnóstico es de gran importancia para el establecimiento de los cursos de acción en estas situaciones. Ante un caso de coma irreversible, con un diagnóstico de tal severidad de daño neurológico surge el planteo de la discontinuación del tratamiento, en especial los de soporte vital, como la asistencia ventilatoria iniciada al nacer. Este tema ha sido, y es motivo de múlti- 
ples preocupaciones y controversias dados los diferentes criterios que se aplican en la toma de decisiones en esta situación especial del comienzo de la vida ${ }^{13}, 14$. Es diferente Ia situación cuando se ha establecido la muerte encefálica. En tal caso, según los criterios estrictos aprobados internacionalmente, el médico no está éticamente obligado a mantener los tratamientos, dado que se establece que él equivale a la muerte de la persona. Así pues, la declaración de muerte ha evolucionado desde cuando se la consideraba a partir de la detención definitiva de la función cardiorrespiratoria. Este cambio en la definición de muerte se ha aceptado desde que la tecnología ha hecho posible sostener esas funciones vitales. Sin embargo hay sociedades y culturas que no aceptan estas apreciaciones científicas, como las del Japón o más recientemente algunas comunidades en los Estados Unidos, que mantienen el criterio de cesación de las funciones cardjorrespiratorias como sinónimo de muerte ${ }^{15}$.

En el caso comentado es no mantener tratamientos incficaces que no tenían justificación en términos de supervivencia. Por lo tanto se realizó lo correcto, no apresurando la muerte y manteniendo las medidas de confort, evitando así el encarnizamiento terapéutico o el tratamiento éticamente desproporcionado. No existio conflicto de principios entre las partes, pues tanto el equipo médico como los familiares observaron el de no maleficencia con respecto a la niña. Dado que la medicina no podría ofrecerle ningún beneficio (por supuesto, luego de tener claro el diagnóstico de la situación clínica), to mejor era no utilizar medios desproporcionados que sólo prolongarian la agonía.

En lo que respecta a las decisiones, en este caso se aceptó que los tesponsables de ello son los padres, de quienes se espera que guíen sus acciones en la perspectiva del mejor interés de su hijo ${ }^{16-18}$. Cumpliendo con la exigencia del consentimiento informado, se permitio la decisión autónoma, luego de una información objetiva y sin ejercer presiones. Se atendieron todos los aspectos humanos relativos a la familia, así como también al equipo tratante. Hubo un equilibrio entre la responsabilidad del médico hacia el paciente basada en su experiencia y competencia, lo que le obliga a un juicio objetivo, y Ia de los padres. Los padres tienen el deber de res- ponsabilidad hacia el niño y su rol en este proceso de toma de decisiones está impregnado de gran estrés emocional. Destacamos la impottancia que tiene la fluidez de la relación médicopaciente-familia, de excelente comunicación y confianza en estas situaciones tan dolorosas para todos los participantes, como lo es la muerte de un recién nacido a la vida.

\section{Referencias}

I. Hartard Medical School: A definition of irreversible coma. Report of the Ad-Hoc Committee of the Harvard Medical School to examine the definition of Brain Death. JAMA 1968: 205: 337-340.

2. Kusthes $T$ : When do organs become "spare parts"? Cambridge Quarterly of Health Care Ethics 1992: 1: 349-353.

3. Siewnu DA. Capron AM. Peacock WJ: The use of anencephalic infants as organ sources: a critique. JANIA 1989: 261: 1773-1781

4. Arrus ID. Shinnal S: Anencephatic newborns as organ donnors: a critique. JAMA 1988; 259: 2284-2285.

5. Arhural $S$ : Brain death in early infancy. J Heart Lung Transplant 1993; 12: \$176-178.

6. Couiter $D L$ : Neurologic uncertainty in newborn in tensive care. NEJM 19B7; 316: 840-844.

7. Truog $R D$ : Is it time to abandon Brain Death? Hastings Center Report 1997: 27: 29-37

8. Wildes KW: Death: A persistent controversial state. Kennedy Institute of Ethics Journal 1996: 6: 378-38I.

9. Pellegrino ED: Clinical Ethics: Balancing Praxis and Theory. Kennedy Institute of Ethics Journal 1996: 6: i47-351.

10. Sass HM: The Clincil as Testing Ground for Moral Theory: A European View. Kennedy Institute of Ethics Journal 1996; 6: 351-355.

11. Astwad $S$ : Brain death determination in infants and children. In Machado. C.(Ed): Brain Death, Amsterdam, Elsevier Science BV 1995; 201-206.

12. Tusk Force. Guideliness for determination of brain death in children. Arch Neurol 1987; 44: 587-588.

13. American Academy of Pediatrics. Committee on Bioethics: Treatment of critically ill newborn. Pediatrics 1983: 72: $565-566$.

14. Vermon $D D$ : Withdrawal of life-support in pediatric critical care. In Machado C (Ed): Brain Death. Amsterdam, Elsevier Science B.V. 1995: 213-221.

15. Child Abuse and Nelect: Prevention and Treatment Program. Federal Register 50: 14878: April 15. 1985.

16. American Academy of Pediaric. Commitiee on Bioethics. Informed consent, pareolal permission, and assent in pediatrics practice. Pedialrics 1995; 95: 314317.

17. Duff RS: Counseling families and deciding care criticalty ill or dying patienls. Pediatrics 1979; 64: 17 . 23.

18. Strong $C$ : The neonatologist's duty to patient and parents. The Hasting Center Report 1984; 14: 10-16. 